\title{
NIL PI-RINGS
}

\section{S. A. AMITSUR}

Let $S$ be a ring which satisfies a polynomial identity (in short: a PI-ring). The ring $S$ will be said to be a PI-ring of degree $d$ if $d$ is the minimal degree of the polynomial identities satisfied by $S$. We denote by $N=N(S)$ the radical of $S$, that is, the sum of all nilpotent ideals of $S$. Levitzki [1] ${ }^{1}$ has proved that a nil PI-ring of degree $d$ is an L-ring (that is, it coincides with its lower radical) and its length is bounded by $\log d / \log 2$. In the present note we show that the length of a nil PI-ring is not greater than 2 and that nil PI-rings of length 2 really exist. Even more, if $S$ is a nil PI-ring of degree $d$ then $S / N$ is a nilpotent ring whose index is bounded by $[d / 2]$. This is a direct consequence of the following generalization of [1, Theorem 1]:

ThEOREM 1. If $S$ is a PI-ring ${ }^{2}$ of degree d and $T$ is a nil subring of $S$, then $T^{m} \subseteq N$ where $m=[d / 2]$.

Proof. First we consider the case ${ }^{3}$ where $T$ is a nilpotent subring of $S$. The proof of this case differs from the proof of [1, Theorem 1] only in that we consider a nilpotent subring instead of a single nilpotent element. That is, we consider the following subrings of $S$ :

$$
\begin{aligned}
A_{2 i-1} & =T^{n-i} S T^{i-1}, \\
A_{2 i} & =T^{n-i} S T^{i},
\end{aligned} \quad i=1,2, \cdots, n,
$$

where $n$ is an integer greater than $m=[d / 2]$.

It is readily seen that $A_{\lambda} A_{\mu} \subseteq S T^{n} S$ if $\lambda>\mu$, hence

$$
A_{i_{1}} A_{i_{2}} \cdots A_{i_{d}} \subseteq S T^{n} S,
$$

if $\left(i_{1}, \cdots, i_{d}\right)$ is a permutation of the $d$ letters $1,2, \cdots, d$ which is not the identical permutation.

By (1) it follows also that

$$
A_{1} A_{2} \cdots A_{d}=\left(T^{n-1} S\right)^{d} T^{m} .
$$

We may assume by [1, Lemma 3$]$ that $S$ satisfies the following identity : 4

Received by the editors June 19, 1950.

${ }^{1}$ Numbers in brackets refer to the bibliography at the end of the paper.

${ }^{2}$ It is not assumed that $S$ is a nil ring.

${ }^{3}$ I am indebted to Levitzki for the present proof of this case.

${ }^{4}$ Compare with (10) of [1]. For the conditions satisfied by the coefficients of the identity see $[1$, p. 335$]$. 


$$
x_{1} x_{2} \cdots x_{d}=\sum \beta^{-1} \beta_{(i)} x_{i_{1}} \cdots x_{i_{d}},
$$

where the sum ranges over all permutations (i) of $d$ letters, except the identical permutation.

From (2), (3), and from condition (II) of [1] satisfied by the coefficients of the identity (1), it follows by substituting $x_{i}=a_{i}$ in (4) where $a_{i}$ ranges over all elements of $A_{i}, i=1, \cdots, d$, that

$$
\left(T^{n-1} S\right)^{d} T^{m} \subseteq S T^{n} S .
$$

Since $T$ is nilpotent, there exists a smallest exponent $n$ such that $S T^{n} S$ is a nilpotent ideal. Suppose $n>m$, then by (5) it follows easily that $\left(S T^{n-1} S\right)^{d+1} \subseteq S T^{n} S$, hence $S T^{n-1} S$ is also nilpotent which is a contradiction to the minimality of $n$. This completes the proof of the theorem in the case of nilpotent subrings.

We turn now to the general case. Let $T$ be a nil subring of $S$. By $[1 \text {, Theorem } 1]^{5}$ it follows that the quotient ring $(T, N) / N$ satisfies an identity of the form $x^{m}=0$, hence $(T, N) / N$ is semi-nilpotent (Kaplansky [3, Theorem 5] and Levitzki [1]). Since $N$ is semi-nilpotent, the subring $(T, N)$ of $S$ is also semi-nilpotent. Let $t_{1}, \cdots, t_{m}$ be any $m$ elements of $T$, then the semi-nilpotency of $T$ implies that the ring $\left\{t_{1}, \cdots, t_{m}\right\}$ generated by these elements is nilpotent, hence by the preceding case $\left\{t_{1}, \cdots, t_{m}\right\}^{m} \subseteq N$. Thus $t_{1} \cdot t_{2} \cdots t_{m}$ $\in N$. Since this holds for any arbitrarily chosen elements of $T$, we have $T^{m} \subseteq N$. q.e.d.

REMARK. By the preceding proof it follows that if $T$ is a nilpotent subring of $S$ of index $p>m$, then $S T^{m} S$ is a nilpotent ideal in $S$. A more detailed application of (5) shows that $1+d+\cdots+d^{p-m}$ is an upper bound for the index of $S T^{m} S$. Indeed by (5) we have $\left(S T^{m} S\right)^{d+1}$ $\subseteq S T^{m+1} S$ and for the same reason $\left(S T^{m+1} S\right)^{d} S T^{m} \subseteq S T^{m+2} S$; hence $\left(S T^{m} S\right)^{1+d+d^{2}} \subseteq S T^{m+2} S$. By a successive application of (5), we obtain

$$
\left(S T^{m} S\right)^{1+d+\cdots+d^{p-m}} \subseteq S T^{p} S=0,
$$

which proves the remark.

By the preceding theorem, we have the following corollary.

CoRollary. If $S$ is a PI-ring of degree $d$ such that its radical $N$ is a nilpotent ideal of index $p$, then the nil subrings of $S$ are nilpotent rings of index not greater than $p[d / 2]$.

REMARK. It has been shown recently [2] that the total matric algebra of order $n^{2}$ over a commutative field is a PI-ring of degree

${ }^{5}$ Apparently, [1, Theorem 1] is a special case of the preceding case of our theorem. 
$2 n$. Hence by the preceding corollary it follows that the nil subrings of such algebras are nilpotent rings of index less than or equal to $n$. This is a special case of the well known result concerning nil subrings of rings which satisfy both chain conditions.

A simple consequence of Theorem 1 is:

Theorem 2. If $S$ is a nil PI-ring of degree d, then $S / N$ is a nilpotent ring whose index is bounded by $[d / 2]$.

This implies that in this case $S$ is a nil PI-ring, $S=N_{2}(S)$, that is:

CoRollaRy. A nil PI-ring is an L-ring of length less than or equal to 2.

We conclude with an example of a nil PI-ring $S$ of degree $2 n$ such that $S / N$ is a nilpotent ring whose index is $n$. This example shows that Theorem 2 provides a complete solution of the problem of the length of nil PI-rings and their structure modulo their radical.

We construct our example as follows: Let $R$ be a commutative ring with a unit such that its radical $N(R)$ is not nilpotent. Denote by $c_{i k}, i, k=1,2, \cdots, n$, an orthogonal base of a total matric algebra $R_{n}$ of order $n^{2}$ over $R$. One can easily generalise [2, Theorem 1] to total matric algebras over commutative rings and thus one obtains the result that $R_{n}$ satisfies a polynomial identity of degree $2 n$ (that is, the standard identity $\left.S_{2 n}(x)=0\right)$. Our required ring $S$ is defined as the totality of the matrices $\sum \alpha_{i k} c_{i k}$ where $\alpha_{i k} \in N(R)$ for $i \geqq k$. No restriction is imposed on the elements $\alpha_{i k}, i<k$, except that $\alpha_{i k} \in R$. Since $S \subseteq R_{n}$, it follows that $S$ is a PI-ring of degree less than or equal to $2 n$. It is readily verified that $S^{n} \subseteq N(R)_{n} \subseteq N(S)$ where $N(R)_{n}$ is the totality of the matrices $\sum \beta_{i k} c_{i k}, \beta_{i k} \in N(R)$. Now consider the element $c=c_{12}+c_{23}+\cdots+c_{n-1 n}$. The ideals $c^{i} S, i=1,2, \cdots, n-1$, contain a ring isomorphic to $N(R)$, that is, the ring of the matrices $c^{i} \bar{r}=\rho c_{11}$ where $\bar{r}=\rho c_{i+1}, \rho \in N(R)$. The latter ring is not nilpotent, hence $c^{i} \notin N(S)$. This implies by [1, Theorem 1] that the degree of $S$ is greater than or equal to $2 n$. This completes the proof that the ring $S$ has the required properties.

\section{BIBLIOGRAPHY}

1. J. Levitzki, $A$ theorem on polynomial identities, Proceedings of the American Mathematical Society vol. 1 (1950) pp. 334-341.

2. S. Amitsur and J. Levitzki, Remarks on minimal identities for algebras, Proceedings of the American Mathematical Society vol. 1 (1950) pp. 449-463.

3. I. Kaplansky, Rings with a polynomial identity, Bull. Amer. Math. Soc. vol. 54 (1948) pp. 575-580.

JERUSALEM UNIVERSITY 